\title{
Intraoperative complications in pediatric neurosurgery: review of 1807 cases
}

\author{
Erik J. van Lindert, MD, PhD, ${ }^{1}$ Sebastian Arts, BSc, ${ }^{2}$ Laura M. Blok, MD, ${ }^{2}$ Mark P. Hendriks, MD, ${ }^{2}$ \\ Luc Tielens, MD, ${ }^{2}$ Martine van Bilsen, MD, ${ }^{1}$ and Hans Delye, MD, $\mathrm{PhD}^{1}$
}

Departments of ${ }^{1}$ Neurosurgery and ${ }^{2}$ Anesthesiology, Radboud University Medical Centre, Nijmegen, The Netherlands

\begin{abstract}
OBJECTIVE Minimal literature exists on the intraoperative complication rate of pediatric neurosurgical procedures with respect to both surgical and anesthesiological complications. The aim of this study, therefore, was to establish intraoperative complication rates to provide patients and parents with information on which to base their informed consent and to establish a baseline for further targeted improvement of pediatric neurosurgical care.
\end{abstract}

METHODS A clinical complication registration database comprising a consecutive cohort of all pediatric neurosurgical procedures carried out in a general neurosurgical department from January 1, 2004, until July 1, 2012, was analyzed. During the study period, 1807 procedures were performed on patients below the age of 17 years.

RESULTS Sixty-four intraoperative complications occurred in 62 patients (3.5\% of procedures). Intraoperative mortality was $0.17 \%(n=3)$. Seventy-eight percent of the complications $(n=50)$ were related to the neurosurgical procedures, whereas $22 \%(n=14)$ were due to anesthesiology. The highest intraoperative complication rates were for cerebrovascular surgery $(7.7 \%)$ and tumor surgery $(7.4 \%)$. The most frequently occurring complications were cerebrovascular complications (33\%).

CONCLUSIONS Intraoperative complications are not exceptional during pediatric neurosurgical procedures. Awareness of these complications is the first step in preventing them.

http://thejns.org/doi/abs/10.3171/2016.3.PEDS15679

KEY WORDS intraoperative; complication; pediatric; neurosurgery; anesthesia

$\mathrm{A}$ CCURATE intra- and postoperative complication rates are indispensable in providing patients and their caregivers with the proper knowledge to submit informed consent prior to surgical procedures. Complications after pediatric neurosurgical procedures (within the first 30 days) are relatively frequent $(16 \%-20 \%) .^{10,25}$ However, very little has been published on complications that occur during pediatric neurosurgical procedures under the shared responsibility of neurosurgeon and anesthesiologist.

Accurate registration of personal and institutional complication rates allows comparison with published case series. Comparison of a physician's or institution's results with the published "standard of care" can reveal shortcomings and areas for improvement. However, literature on intraoperative complication (IOC) rates is so sparse that this benchmarking is not possible. Therefore, IOC rates may be acquired as reference values within an institution as a baseline for further targeted improvement, although the impact of such complication registration on quality improvement remains to be established. ${ }^{8,14}$

A major reason to look at complication rates is that they offer the opportunity for commonalities to emerge. Complications that have been dismissed before as incidental or nonsignificant may now be recognized as structural complications that might be solved (and intraoperative complications belong to this group because of their rareness). Therefore, complication registration is a prerequisite for improvement of neurosurgical care.

The treating surgeon and other staff members of the same department, but not physicians from other disciplines, usually register the complications. Intraoperative complications coming to the attention of the anesthesiologist may go unnoticed by the surgeon, especially if they are archived in an anesthesiological database. Some of these complications may occur during phases of the surgery in which the surgeon is not in the operating theater but may affect surgery and surgical outcome. The re-

ABBREVIATIONS IOC = intraoperative complication.

SUBMITTED November 19, 2015. ACCEPTED March 28, 2016.

INCLUDE WHEN CITING Published online May 27, 2016; DOI: 10.3171/2016.3.PEDS15679. 
sults of prospective surveillance of complications were published in an article by Drake et al., ${ }^{10}$ and that study constituted the first report on complications in pediatric neurosurgery, but the authors did not mention intraoperative or anesthesiological complications separately, and whether anesthesiological complications were collected in that study remains unknown. As a follow-up, the Canadian Pediatric Neurosurgery Study Group (CPNSG) tried to define complications on a consensus basis for pediatric neurosurgery; however, they did not address intraoperative complications. ${ }^{11}$ Our previous study on pediatric neurosurgical complications occurring within 30 days of surgery in a series of 1000 procedures was the first to mention intraoperative complications separately, including anesthesiological complications. ${ }^{25}$ In addition, many intraoperative surgical complications may require the anesthesiologist to intervene, whereas anesthesiological complications may be a reason to postpone or abort surgery. Therefore, we see good reasons to draw extra attention to complications occurring intraoperatively.

To qualitatively establish the different types of intraoperative complications, as well as our intraoperative complication rates, we extended our previously published study and conducted a retrospective analysis of our clinical neurosurgical and anesthesiological complication databases.

\section{Methods \\ Study Design}

This study, which was approved by the local institutional review board, was a retrospective single-center review of a clinical complication registration database of a consecutive pediatric patient cohort with prospective data collection. It was performed in an academic hospital serving a population of 1.7 million for neurosurgery; however, for pediatric neurosurgery, the hospital served a population of 3 million. The reviewed complications occurred in pediatric neurosurgical procedures performed from January 1, 2004, until July 1, 2012. During most of this period, the staff of the neurosurgical department consisted of 7-9 neurosurgeons, one of whom was dedicated to pediatric neurosurgery. After 2010, a second pediatric neurosurgeon joined the pediatric service. All staff members participated in the surgical treatment of children. However, elective surgery on children was planned preferably, and performed predominantly, by the pediatric neurosurgeons. From 2005 on, the majority of children were anesthetized by a qualified pediatric anesthesiologist. According to hospital rules, all children younger than 16 years of age undergoing major or elective surgery or with comorbidity and American Society of Anesthesiologists (ASA) class > II should be anesthetized by a pediatric anesthesiologist. In the case of relatively minor emergency surgeries in ASA Class I and II children, a general anesthesiologist may perform the anesthesia. All procedures were carried out under general anesthesia. Anesthesia was induced with an intravenous anesthetic; small children underwent mask induction with sevoflurane. Anesthesia was maintained with opioids, a volatile anesthetic, and muscle relaxants.

\section{Patients and Setting}

All patients under the age of 17 years who underwent neurosurgical procedures at our institution were included in this study. All operations were performed at Radboud University Medical Center.

\section{Data Collection}

For each patient, demographic and clinical data were prospectively collected in Microsoft Excel by the first author (E.J.V.L.). All adverse events, defined as adverse events occurring during the surgical procedure from administration of local or general anesthesia to extubation and/or transport from the operating room observed by any member of the operating team within this period, no matter how small, were registered during surgery or later retrieved from operative charts. Complications discovered later were registered as postoperative complications. Data from a separate anesthesiological database (NOVAS, Philips) were included post hoc to complete the database. Diagnoses in main groups and subgroups, type of operative procedure, and intra- and postoperative complications were registered, as was the name of the operating surgeon. Types of complications were not classified or defined before starting the registration. Comorbidity and risk factors were not assessed separately. Intraoperative complications were reviewed post hoc and divided into several subgroups based on surgical indication, also by the first author (E.J.V.L.) (Table 1).

Hemodynamic/cerebrovascular complications were defined as complications related to vascular damage, blood flow, blood pressure, and/or cardiac function. Intraoperative hemorrhage was defined as unexpected excessive blood loss resulting in the need for transfusion, cardiovascular stabilization, or premature termination of the procedure because of poor visualization. ${ }^{1,2}$ All hemodynamic complications resulting from surgical blood loss or directly from surgical manipulation of neural tissues (e.g., brainstem) were considered neurosurgery-related. All hemodynamic complications that were caused by administration of medication, occurred spontaneously because of the underlying disease, or remained unexplained were considered anesthesiological. Intraoperative CSF leaks were reported in extradural surgeries in which dura mater was opened unintentionally, for example, craniosynostosis surgery.

The drain/catheter subgroup of complications concerns mainly difficulty in positioning the drain/catheter. The neural injury subgroup comprises such injuries to the brain, cranial and peripheral nerves, and spinal cord that were unintended and not part of the planned surgery (e.g., corticotomy, removal of a cerebellar tonsil) and not the result of normal neural tissue manipulation and, therefore, constituted an intraoperative notice of unintended structural damage, independent of observed postoperative neurological deterioration. Skull fractures by skull clamp were collected separately. The miscellaneous subgroup involves complications that could not be classified in one of the other subgroups. All anesthesia-related complications were placed in the anesthesia subgroup and further subdivided into hemodynamic complications, hypothermia, hypoxemia, allergic reactions, and pulmonary complications. 
TABLE 1. Intraoperative complication rates for different indications for surgery

\begin{tabular}{lrcc}
\hline \multicolumn{1}{c}{ Indication } & Total No. & No. of IOCs & \% IOCs \\
\hline CSF disturbance & 804 & 18 & 2.2 \\
\hline Congenital disorder & 308 & 12 & 3.9 \\
\hline Brain tumor & 284 & 19 & 6.7 \\
\hline Trauma & 148 & 5 & 3.4 \\
\hline Infection & 42 & 0 & 0 \\
\hline Functional & 40 & 0 & 0 \\
\hline Cerebrovascular & 39 & 3 & 7.7 \\
\hline Spinal tumor & 25 & 2 & 8 \\
\hline Peripheral nerve & 5 & 0 & 0 \\
\hline Degenerative disease & 4 & 1 & 25 \\
\hline Miscellaneous & 108 & 4 & 3.7 \\
\hline Endoscopic & 225 & 8 & 3.6 \\
\hline Nonendoscopic & 1582 & 56 & 3.5 \\
\hline Total & 1807 & 64 & 3.5 \\
\hline
\end{tabular}

\section{Results}

A total of 1807 pediatric neurosurgical procedures were performed in 1148 patients from January 1, 2004, through July 1, 2012. Of the 1807 procedures, 977 (54\%) were performed by the 2 dedicated pediatric neurosurgeons. The other 830 procedures were performed by other neurosurgeons or by residents. The patients' median age was 72 months (6 years, range 1 day to 16.9 years). On average, 1.6 operations were performed per patient within this period. Table 1 lists the different surgical indication groups. Endoscopic surgery was performed in 225 procedures (12.5\% of all procedures).

Sixty-four intraoperative complications (3.5\% of procedures) occurred in 62 patients (5.4\% of patients). The IOC rates were highest in surgery for spinal tumors, cerebral tumors, and cerebrovascular disease, and low during surgery for CSF disturbances (Table 1). The IOC rates for endoscopic and nonendoscopic procedures were similar $(3.6 \%$ vs $3.5 \%)$ (Table 1$)$.

The mean age of patients who had an IOC was 5.7 years; 19 of these patients (30\%) were 1 year of age or younger. The whole population had a mean age of 6.0 years; 605 patients were 1 year or younger (33\%).

Seventy-eight percent of complications $(n=50)$ were related to the neurosurgical procedure itself, whereas $22 \%$ (n $=14$ ) were attributed to anesthesia. Therefore, an anesthesiological complication occurred in $0.8 \%$ of all pediatric neurosurgical procedures. Fifteen surgical complications (30\%) required an intervention by the anesthesiologist during surgery. Overall, cerebrovascular/hemodynamic complications were the most frequent, occurring in $1.1 \%$ of all procedures.

\section{Neurosurgical Complications}

Twenty-one surgical cerebrovascular complications occurred; 20 were bleeds, and 1 was a vascular occlusion (Table 2). In 4 cases, complications led to premature termination of the procedures.
TABLE 2. Different types of complications

\begin{tabular}{cc}
\hline Type of Complication & No. of Cases \\
\hline Neurosurgical & 47 \\
\hline Vascular/hemodynamic & 21 \\
\hline CSF leak/dural tear & 7 \\
\hline Drain/catheter related & 8 \\
\hline Skull fracture & 3 \\
\hline Neural injury & 2 \\
\hline Miscellaneous & 6 \\
\hline Anesthesiological & 14 \\
\hline Cardiovascular/hemodynamic & 3 \\
\hline Hypothermia & 2 \\
\hline Hypoxemia & 2 \\
\hline Respiratory & 4 \\
\hline Allergic reaction & 1 \\
\hline Miscellaneous & 2 \\
\hline Intraoperative death & 3 \\
\hline Total & 64 \\
\hline
\end{tabular}

Diffuse bleeding from the tumor bed leading to hemodynamic instability and requiring intraoperative blood transfusion during tumor resection was the most frequent complication $(\mathrm{n}=5)$. In 2 of these cases, surgery had to be terminated prematurely. A lesion to a dural sinus occurred in 3 patients (in a transverse sinus in 1 and in a sagittal sinus in 2) without hemodynamic consequences, whereas opening of a sinusoidal dura of the posterior fossa in 1 infant led to profuse blood loss and asystole requiring resuscitation. The latter patient did not suffer serious consequences, and surgery continued after stabilization. Three catheter placements caused hemorrhagic complications with intraventricular bleeding. In one of the three, a shunt revision, removal of the ventricular catheter led to choroid plexus bleeding; an external ventricular drain had to be placed, the procedure was terminated, and the patient underwent surgery the next day. Two serious venous plexus bleeds occurred during spinal procedures: one led to prolonged surgery, and the other procedure had to be abandoned.

Arterial lesions occurred in 3 patients. One patient had a vertebral artery lesion and suffered a 4.5 -L blood loss caused by a malpositioned screw in dorsal cervical fusion surgery. The second arterial lesion involved a lenticulostriate artery, which was torn out of the middle cerebral artery during craniopharyngioma surgery, leading to clipping of the parent vessel and consequent cerebral ischemia with neurological deficit. The third arterial lesion was a posterior inferior cerebellar artery lesion leading to obliteration of the artery with subsequent cerebellar infarction. One endoscopic procedure (endoscopic third ventriculostomy) led to intraoperative hemorrhage that required placement of an external ventricular drain. In a scoliosis surgery, a hemothorax causing $900 \mathrm{ml}$ of blood loss and requiring thorax drainage occurred during opening of the diaphragm. One cerebellar arteriovenous malformation (previously embolized) caused $2 \mathrm{~L}$ of blood loss in a 
2-year-old; that surgery was halted several times because of hemodynamic instability and could not be continued until stabilization was achieved.

During endovascular treatment of an arteriovenous malformation (performed by a cerebrovascular neurosurgeon), a basilar artery tip thrombosis occurred and was effectively treated with heparin and thrombectomy without consequences.

Unwanted dural lesions occurred in 7 patients. In 5 patients diagnosed with craniosynostosis, dural lacerations occurred. Three of these patients were operated on using an open remodeling technique, and 2 were operated on endoscopically. All dural openings were directly sutured without sequelae and did not lead to longer procedure times. Two CSF leaks during transsphenoidal surgery required an autologous fat graft and placement of an external lumbar drain, which prolonged the procedure time by 20 minutes.

Intraoperative complications related to drains and catheters occurred in 8 procedures. In 6 cases, the complication was related to failure to adequately position a drain in a ventricle. In 1 case, an external ventricular drain was uncoupled by accident without being immediately noticed, leading to significant CSF loss into the bed. Another case was due to a malpositioned ventriculoatrial drain, with the distal catheter in the subclavian artery and inability to correct the position into the right atrium. These drain-related complications affected the duration of surgery, but otherwise had no consequences for anesthesia.

Traumatic injuries caused by use of a Mayfield head skull clamp occurred in 3 patients (aged 2, 7, and 11 years), all were operated on in the prone position for posterior fossa tumors. In 1 case, a supratentorial epidural hematoma was observed as posterior fossa crowding during medulloblastoma resection and required termination of the tumor resection, CT scanning, and surgical management of the hematoma. Twice a small skull impression fracture was observed during application of the Mayfield head holder and required repositioning, without further consequences for the patient.

Neural injuries, recognized as such already during surgery, occurred in 2 patients: a fornix lesion and an olfactory nerve lesion. Both patients had no clinically recognizable postoperative deficits. Our definition of neural injury is rather arbitrary and may have led to underreporting.

There were various complications in the miscellaneous group. The serosa of the intestine was damaged during placement of a ventriculoperitoneal shunt and had to be stitched by the pediatric surgeon consulted. Blisters occurred on 1 patient's buttocks because of excessive iodination and subsequent reclining on a wet rubber mattress. The subcutaneous electrode of a patient with a bilateral cochlear implant was damaged during the skin incision for open brain biopsy. A stereotactic biopsy performed for a pineal region tumor was negative (no representative material); accordingly, reoperation was necessary. During reoperation of the rapidly growing glioblastoma, severe cerebral edema occurred and the procedure had to be terminated before tumor resection could be performed. The patient died 5 days after surgery.

A patient with diffuse craniospinal leptomeningeal tumor dissemination underwent a thoracic laminectomy for open intradural biopsy. The opening of the dura with loss of CSF at the T-2 level was complicated by bradycardia. Under the presumption of tonsillar herniation in the crowded posterior fossa, the patient was placed in an almost vertical head-down position, after which she recovered and stabilized. Surgery was continued, and a biopsy successfully obtained.

Three patients died during surgery. All were trauma patients with severe diffuse cerebral edema. Two patients died of circulatory arrest/asystole, and the third patient died of cerebral herniation while the surgeon attempted to gain access to the ventricles for CSF drainage.

\section{Anesthesiological Complications}

Severe laryngospasm occurred in 3 patients: one patient had an allergic reaction to medication (classified as such), the second had underlying tracheomalacia (Table 2). Hypoxemia at the end of surgery occurred in $2 \mathrm{CSF}$ shunting procedures (involving 3- and 4-month-old infants). In 1 case of bronchial intubation, the hypoxemia was resolved by slightly withdrawing the tube to a correct position. In the other case, the origin of desaturation down to $38 \%$ remained unclear, but was resolved quickly nevertheless. Atelectasis caused by bronchial intubation occurred in one patient and led to desaturation during tumor surgery. Aspiration during anesthesia induction occurred only once and was without consequences for the subsequent short-duration shunt surgery.

Hypothermia occurred in 2 patients and required prolonged anesthesia to rewarm the patient. One child, a 3-month-old with Walker-Warburg syndrome who was in poor neurological condition, was receiving a shunt for hydrocephalus. In this patient, difficulty was encountered in the insertion of lines; intubation was not possible and was converted to a laryngeal mask. As a consequence, anesthetic preparations required 54 minutes. The procedure was prolonged overall, and temperature dropped to $28^{\circ} \mathrm{C}$. The other case was a 16-year-old boy who had a spontaneous cerebellar hematoma with decortication symptoms. His rectal temperature dropped to $23^{\circ} \mathrm{C}$ during ambulance transport, transport to the emergency room, CT scan, and ultimately the operating room. Hemodynamic instability and the need for urgent intervention precluded warming up of the patient. Despite successful surgery, the patient died of cerebral ischemia 2 days later.

A 2-day-old child, operated on in the prone position because of a traumatic subdural hematoma, suffered prolonged hypotension after clot evacuation not caused by blood loss. An attempt to insert an arterial line into the femoral artery caused a thrombosis of the same artery, requiring prolonged anti-platelet aggregation medication.

In the case of an 18-month-old child with a left temporoparietal malignant ependymoma with severe brain swelling, the opening of a tumor cyst caused a sinus tachycardia of $180 \mathrm{bpm}$, which stabilized spontaneously, but shortly thereafter the rhythm changed to severe bradycardia without cardiac output occurred; this change could not be directly attributed to surgery or blood loss during surgery. This resulted in resuscitation; after stabilization, both of the patients' pupils were dilated and the surgery 
was terminated. The child died 2 days later of anoxic encephalopathy.

A subcutaneous extravasation of thiopental in the hand of a 9-year-old boy required intervention by a plastic surgeon. A medication failure (overdosage) occurred in a 4 -year-old girl during shunt revision surgery but had no consequences.

\section{Discussion}

In pediatric neurosurgery, the assessment of adverse events and risk factors for morbidity and mortality is rare, and little is known about the complications of surgical events. The literature on the topic is sparse.

There is still no consensus or standardized guideline on what a complication or an adverse event is. This shortcoming hampers comparison of outcome data among different centers and therapies over time. ${ }^{25}$ Even an apparently simple complication, such as surgical wound infection, was found to have 41 different definitions and 13 different surgical grading scales in 82 studies. ${ }^{7}$ In addition, an event may be a complication in a specific procedure, but not in another, and multiple complications may be difficult to classify correctly, unless the complications are fully independent and not part of the same chain of events. ${ }^{25}$ At least it can be agreed that an adverse event is characterized as untoward, undesirable, or detrimental; having an impact on the patient; and being caused by a health care process rather than the natural course of the disease.?

We defined a complication as "any adverse event," but this is not a clear-cut definition and quite often is relatively arbitrary. A skull fracture caused by the Mayfield head holder is self-evident, but when does blood loss become a complication? At what temperature is hypothermia a complication, and which saturation is required to register hypoxia as a complication? A drawback of our registration is that we did not define these complications before starting and registered only those events that were registered by the treating physicians as a complication or adverse event. Nevertheless, every effort should be taken to register these adverse events also, to learn how to prevent them.

We counted 64 IOCs (3.5\% of procedures) in a period of 8.5 years. More than 20 surgeons (staff and residents) and more than 50 anesthesiologists (also staff and residents) were involved in all neurosurgical procedures in this period. Many IOCs were unique, and those that were not unique occurred only once or occasionally to the individual surgeon or anesthesiologist during the study period. Nevertheless, lessons may be learned even by those not confronted with the IOC, to prevent that same IOC in the future. Therefore, these IOCs are extensively discussed in our institutional morbidity and mortality conferences.

There is no consensus in the literature on the definition of anesthesia-related mortality or morbidity as separate from surgery-related mortality or morbidity. ${ }^{9}$ In many cases, both surgical and anesthesiological factors contribute to the adverse outcome, and thus, separation of the two is partly artificial. However, in many neurosurgical articles in which surgical complications are mentioned, purely anesthesiological complications, such as laryngospasm, are not counted, and in the anesthesiology literature, typical surgical problems are not mentioned. To our knowledge, our study is the first in the neurosurgical literature to make an effort to combine the 2 types of complications. Therefore, we find it helpful to try to define each team member's role in such adverse events to prevent them and improve procedures.

In our series, we had 3 intraoperative fatalities (16.6 per 10,000). All of the patients who died during surgery had high-impact/high-velocity cranial and cerebral trauma with severe brain swelling and underwent emergency surgery for acute brain herniation. In all 3 cases, the patients died of disease rather than surgical or anesthesiological adverse events that, after thorough multidisciplinary evaluation, were considered unpreventable. Nevertheless, we find it necessary to list these as IOCs to encourage thorough investigation of the events and facilitate comparison with others.

\section{Neurosurgical IOCs}

Of all the IOCs in our analysis, 50 (78\%) were related to the surgical procedure or primary disease itself. Little has been published on the incidence of intraoperative complications in the neurosurgical literature. ${ }^{10,25}$ To our knowledge, there are only 2 reports of complications in pediatric neurosurgery, only one of which (our previous study $^{1,2}$ ) mentions intraoperative complications separately.

Aleksic et al. ${ }^{2}$ reported a $9.6 \%$ complication rate in more than 700 pediatric neurosurgical procedures, but considered only anesthesiological complications without mentioning surgical complications.

Although overall IOC rates are almost entirely absent, rates for specific procedures are mentioned occasionally. For example, pediatric severe spinal deformity surgery has a very high IOC rate, with a complication occurring in almost half of the procedures; it was the highest rate we found..$^{19}$

Excessive bleeding is not uncommon in the operating room, and some type of vascular damage is unavoidable when performing surgery. This kind of complication was the most frequent type of IOC in our study, accounting for one-third of all IOCs. However, at what level is blood loss called a complication? We used a practical though arbitrary definition: any blood loss that hampers adequate continuation of surgery or that requires any form of intervention by the anesthesiologist is an IOC. Any damage to a dural sinus or to major arteries or unintended obliteration of these is also considered an IOC.

Skull fractures and epidural or subdural hematomas caused by a 3-pin skull clamp were found to occur in $0.65 \%-1.1 \%$ of children that underwent head fixation. ${ }^{18,26}$ Many of these may be discovered only after surgery and thus are categorized as postoperative complications. However, in our series, all clinically relevant trauma caused by the Mayfield head holder was diagnosed during surgery, and we never diagnosed such a complication in the days following surgery. There is no guideline that describes from which age this head fixation can be used and what pressure can be applied. ${ }^{5}$ Most prone to this complication are patients with thinned skulls caused by chronic increased intracranial pressure, for example, patients with tumors with chronic obstructive hydrocephalus, and pa- 
tients placed in the prone position for surgery on the posterior fossa. ${ }^{5,26}$ Our guideline is to avoid this head holder for children under 2 years of age, to minimize pressure, to study skull thickness on scans and look for the thickest parts of the skull during application avoiding the temporal fossa, and to use a bean bag as much as possible for surgery in the supine position.

The placement of a ventricular drain has always been a freehand technique, although several different guidance techniques have been developed. It is considered an easy procedure, quite often performed by those in training. However, in at least half of the cases, the tip of the catheter is not found in the expected position, and in 10\%-20\% of the cases, it is in brain parenchyma. ${ }^{1,15,24}$ Typically, on average, 2 passes are required to enter the ventricles. ${ }^{15}$ Ventriculostomy-related hemorrhage occurs in $5.7 \%-7.1 \%$ of patients; the rate of clinically significant hemorrhage has been reported as $0.61 \%$, with most cases not being noticed during surgery. ${ }^{6,21}$

It is possible to further analyze our procedure subgroups and compare them with published series of treatments for specific diseases. However, this is vulnerable to different types of bias. First, these published series usually represent studies from specialized centers with sufficient caseloads of the specific disease being reported as well as special interest and expertise. Second, the reported complication rates are often by-products of reporting treatment results. Especially in retrospective studies, it is questionable whether complications have been registered adequately. Third, the special attention that is given to a group of patients under study may result in a lower complication rate, whereas at the same time, complications may be underestimated in patients undergoing routine procedures at the same institution. ${ }^{25}$

We did not try to look into differences between pediatric and adult neurosurgeons with respect to IOCs. First, the number of IOCs is so low that significant comparison is not possible with these numbers in the subgroups. Second, pediatric and adult neurosurgeons operated on different patients and diseases. For example, all craniosynostosis surgeries, all dysraphism surgeries, and $80 \%$ of oncological surgeries are performed by pediatric neurosurgeons. Only the group of CSF disorders, for which adult neurosurgeons performed a substantial number of procedures, is of significant size. But also in this group, the pediatric neurosurgeons operated on the more difficult cases or those patients prone to complications (premature and newborn infants, complex hydrocephalus cases). Case mix correction would make the subgroups so small that significant differences could not be discerned.

With an average of 7 intraoperative neurosurgical complications (range 4-11) per year during the study period, we were not able to identify a possible learning effect and consequent decrease in the IOC rate over time due to the small size of the numbers involved.

\section{Anesthesiological Complications}

Overall, perioperative and anesthesia-related mortality is 10 -fold higher in pediatric patients than in adults, and within this group, it was higher in neonates and infants than in older children. ${ }^{2,4,14,23}$ In developed countries, the perioperative mortality rate is $0.42-6.8$ per 10,000 anesthetics. ${ }^{14}$ Most of the fatalities are associated with airway or cardiocirculatory events. The major risk factors are age $<1$ year, ASA class $\geq 3$, and emergency surgery, general anesthesia, or cardiac surgery. ${ }^{4}$

Studies have indicated that the risk of complications is quadrupled if anesthesiological residents administer the anesthesia or if the resident/anesthesiological nurse is being supervised by an anesthesiologist who supervises multiple procedures. ${ }^{17}$ Moreover, complications in children occur less frequently when anesthesia is induced by an experienced pediatric anesthesiologist than when it is induced by a general anesthesiologist. ${ }^{16,17}$ Also, the experience of the anesthesiologist has an effect on the occurrence of complications. Auroy et al. ${ }^{3}$ state that a minimum caseload of 200 pediatric general anesthesia procedures per year is necessary to reduce the incidence of complications and improve the level of safety in pediatric practice.

As with mortality, the complication rate in general has been reported to be higher in infants than in older children., ${ }^{4,20,23}$ This was not confirmed in our study. One-third of the IOCs occurred in children $\leq 1$ year, but this group also constituted one-third of all patients. Most likely our study is different because surgical complications outnumbered anesthesiological complications. However, half of the anesthesiological complications occurred in infants younger than 3 months of age.

In our study, the respiratory complications consisted of laryngospasm, atelectasis, and aspiration. Laryngospasm is the main cause of anesthesia-induced circulatory arrest in children. It occurs mostly during the emergence from anesthesia and is provoked by a lesser depth of anesthesia. ${ }^{4}$ Oxygen reserve capacity and apnea tolerance are low in newborns and infants, meaning that hypoxemia may occur rapidly and may lead to bradycardia. ${ }^{4}$ Another explanation is that anesthesia is often induced in pediatric patients while they are sitting on a parent's lap, and after induction the patient is transferred to the operating table, which may awaken the patient or lighten the state of anesthesia and increase the risk of laryngospasm. ${ }^{23}$ Even though laryngospasm often occurs during the induction of or emergence from anesthesia, the majority of anesthesiological complications occur during the maintenance phase of anesthesia $(80.6 \%){ }^{23}$ Thus, it is not the hectic induction and emergence that are responsible for most complications, but the seemingly quiet period in between. Therefore, vigilance during the maintenance period is essential to reduce complications and increase the safety of patients. The anesthesiological literature does not indicate whether these problems are caused primarily by surgical problems. In our study it was clear that hemodynamic and cardiovascular problems, in the majority, were the result of surgical blood loss or had a neurological cause.

Our rate for anesthesia-related complications was $0.89 \%$. In the pediatric anesthesiological literature, the rate of anesthesiological complications is $2.8 \%-9.6 \%{ }^{2,12,23}$ Tay et al. reported a $3.4 \%$ IOC rate in their neurosurgical subgroup, whereas the IOC rate was $2.8 \%$ for all pediatric anesthesiological procedures. It is possible that what we counted as a neurosurgical complication was counted as an anesthesiological complication in that study (especially 
hemodynamic complications with a surgical cause). Only 1 study has reported solely anesthesiological complications in a pediatric neurosurgery patient cohort. In a retrospective study over the period 1996-2000, Aleksic et al. ${ }^{2}$ reported a $9.6 \%$ IOC rate, with surgery duration greater than 240 minutes, surgery in the sitting position, and comorbidity as special risk factors. Major reasons for this higher rate may be that in their series, the anesthesiological procedures were all performed by general anesthesiologists, and their study is from a much earlier time period. Comparison of studies, however, is not possible.

Most likely, cardiovascular and respiratory complications were underreported in our study, because we did not define these strictly before starting registration. When, for instance, cardiovascular complications were sharply defined as $20 \%$ deviations of the measured variables from baseline values, then IOC rates of $11 \%-22 \%$ for these complications (brady- and tachycardia, hypo- and hypertension) were described in a pediatric neuroendoscopic series. $^{22}$ The same will hold true for hypothermia and hypoxemia. Therefore, definition of these complications is required if we want to continue with a more reliable complication registration.

\section{How to Improve and Lessons Learned}

Our study was prospective in counting complications but retrospective in the analysis of adverse events. Analysis during morbidity and mortality conferences relies on postevent reconstruction of adverse events, with an attempt to recreate events through a review of hospital records and interviews of involved staff when possible, but often without first-hand knowledge of the events. This leads to a lack of necessary information on the processes of care that resulted in the outcome or the context in which a specific event occurred. Christian et al., ${ }^{8}$ in a prospective study, observed 10 general surgery procedures and found critical system features that influenced patient safety in all 10 cases. Communication breakdown and information loss, as well as increased workload and competing tasks, posed the greatest threats to patient safety in the operating room. This reflects the popular wisdom that medical error is usually caused by system and communication problems.

Work flow and procedures have been improved in our hospital by the introduction of a briefing with the complete operating room team at the start of the day of surgery, a time-out procedure before each surgery, a sign-out procedure after each surgery, a double-check procedure for medication and blood products, and a debriefing at the end of the workday. These procedures have been further improved and tightened by digitalization of these processes with the introduction of new electronic health record software (EPIC Systems). Furthermore, in 2013, all personnel involved in neurosurgical procedures received crew resource management (CRM) training to improve communication in the operating room and to increase safety; yearly refresher courses are planned. The refresher courses also serve to improve the procedures that are used collectively.

In contrast, however, Fabri and Zayas-Castro ${ }^{13}$ provocatively concluded that it is human error, not communication and systems, that underlies surgical complications. In a prospective study, they created and validated a system for classifying medical errors and found that errors in surgical technique $(63.5 \%)$, judgment errors $(29.6 \%)$, inattention to detail $(29.3 \%)$, and incomplete understanding $(22.7 \%)$ were the main contributors to surgical complications. This is rather concerning and a reason for all involved staff to focus on every single adverse event. The attitude of "this adverse event can happen to anybody, bad luck" should be reversed into "this adverse event should not happen ever again to me or to us."

Because many IOCs are incidental, it is difficult to develop an explicit strategy for their prevention. The mainstay now is to carefully register IOCs and analyze and discuss them during (multidisciplinary) morbidity and mortality conferences. In our monthly morbidity and mortality conferences, at which we discuss all complications, we always try to focus on at least 1 complication and draw lessons from it with direct implications for daily clinical use. This may take the form of advice or a new house rule or clinical guideline. This procedure is, as a rule, easier for the more frequent postoperative complications, for which a shunt infection prevention protocol, a spine instrumentation infection prevention protocol, and the use of navigation for shunts as a house rule are examples of improvement.

The complications listed in Table 2 suggest that several complications may be preventable. We adapted several procedures and protocols as a result.

Severe blood loss cannot always be prevented, but several measures may help to prevent severe hemodynamic problems. The liberal use of tranexamic acid has been introduced for those cases in which severe blood loss is anticipated or, when it occurs during surgery, to improve hemostasis. When performing surgery around a venous sinus or when opening the dura of the posterior fossa with a persistent major occipital sinus in an infant, the anesthesiologist is on standby to deal with significant blood loss in a short period. Communication between neurosurgeon and anesthesiologist during anticipated critical phases of a surgical procedure is increased so that both can be better prepared. If necessary, surgery is halted for a period so that the anesthesiologist can catch up with the blood loss and stabilize hemodynamics and/or coagulation. Use of more expensive hemostatic agents such as the Floseal hemostatic matrix (Baxter) has increased despite budgetary restrictions.

Surgical hemostasis and blood replacement management in craniosynostosis surgery has been reviewed and given more attention to reduce the transfusion rate.

The application of the Mayfield head holder has received more attention to prevent skull fractures and hematomas. The Mayfield will not be used in children under 2 years of age, and its use may be avoided or the applied pressure reduced in children older than 2 years with chronic hydrocephalus and reduced skull thickness. In addition, we acquired a pediatric head holder that uses gel pads instead of skull pins.

In patients with small ventricles and/or displaced intracranial anatomy who require a ventriculostomy, the use of neuronavigation has now become obligatory to prevent a dry tap or incorrect drain positioning.

During skin preparation with iodine solution or chlor- 
hexidine, care is taken not to spill the solution, which would wet the pad on which the patient is lying and could cause blisters.

We have become more restrictive toward patients who have an upper airway infection within 2 weeks before elective surgery. Surgery is postponed in these cases if possible.

Communication between surgeon and anesthesiologist is intensified during all phases, especially positioning of the patient. As an example, the surgeon asks the anesthesiologist to check on tube depth after flexing the head of the patient.

\section{Limitations of our Study}

When starting our clinical complication registration database, we did not define or characterize "complication," although main groups and subgroups were defined. Because we retrospectively analyzed a clinical database and did not perform a prospective study, validation of complications was not performed. Although some complications are unequivocal (e.g., skull fracture caused by a Mayfield head holder), others were very subjective in nature (e.g., unexpected blood loss). The most frequent IOC, vascular damage/hemorrhage, is also the most ill-defined and arbitrarily assessed IOC. Bleeding is inherent in every surgery to a certain extent; even postoperative bleeding is accepted as normal and is why drains are used. Nevertheless, bleeding is also accepted as a complication if it is more than anticipated, unexpected, the result of a technical error or bad judgment, and/or leads to extra risks for a patient, the need for blood transfusion, abortion of surgery, and so on. It is practically impossible to define the boundary between "normal" hemorrhage and "hemorrhage as a complication." A mass transfusion is accepted as normal when operating on a glomus tumor but not in hydrocephalus surgery. Severe hemorrhage from a venous sinus when removing a tumor from the wall of the sinus is not a complication, but the expected result of a planned maneuver, whereas a little blood loss from an accidentally opened sinus might be the result of inferior surgical technique and, thus, a complication. Whether this last example should be addressed as a surgical technical problem or as a complication can be discussed, because most likely the patient will not suffer from it. Nevertheless, it can become the reason for prolonged surgery, unnecessary blood transfusion, or postoperative hemorrhage, placing the patient at risk.

As another example, an unintended fornix transection during endoscopy may not be clinically significant. This, however, is by sheer luck. The "mistake" may not be noticed; however, nothing will be learned if only complications that lead to persisting deficits are recorded. The fornix lesion should therefore be regarded as an adverse effect, as it is unintended structural damage to the patient that is, as a rule, preventable.

Therefore, we suggest that these types of problems, called technical errors or problems by some, be registered as complications. The same argument, though to a lesser extent, is applicable to other complications as well.

Because of the subjective nature of the definitions we used for complications and also because external validation was not performed (we retrospectively studied a clinical database), some complications may not have been recognized and/or recorded in medical charts when they occurred, and thus the actual IOC rate may be higher than reported. Our study should therefore not be considered an accurate quantitative account of IOC rates that can be used for benchmarking or comparative studies. Rather, it should be considered a qualitative study undertaken to establish the urgency of the problem, to gain some insight into the different problems that may occur during surgery, and to define types of complications, as well as areas for improvement. Therefore, our study is meant to inspire further research into the topic of IOCs in pediatric neurosurgery.

\section{Conclusions}

Intraoperative complications during pediatric neurosurgical procedures are not rare. However, many complications are incidental. Awareness of these possible complications is the first step in preventing them. Complication registration is thus essential and a prerequisite for risk reduction. It should also be emphasized that IOC registration is a shared responsibility for both neurosurgeons and anesthesiologists.

\section{References}

1. Abdoh MG, Bekaert O, Hodel J, Diarra SM, Le Guerinel C, Nseir R, et al: Accuracy of external ventricular drainage catheter placement. Acta Neurochir (Wien) 154:153-159, 2012

2. Aleksic V, Radulovic D, Milakovic B, Nagulic M, Vucovic $\mathrm{D}$, Antunovic V, et al: A retrospective analysis of anesthesiologic complications in pediatric neurosurgery. Paediatr Anaesth 19:879-886, 2009

3. Auroy Y, Ecoffey C, Messiah A, Rouvier B: Relationship between complications of pediatric anesthesia and volume of pediatric anesthetics. Anesth Analg 84:234-235, 1997 (Letter)

4. Becke K: [Complications in pediatric anesthesia.] Anaesthesist 63:548-554, 2014 (Ger)

5. Berry C, Sandberg DI, Hoh DJ, Krieger MD, McComb JG: Use of cranial fixation pins in pediatric neurosurgery. Neurosurgery 62:913-919, 2008

6. Binz DD, Toussaint LG III, Friedman JA: Hemorrhagic complications of ventriculostomy placement: a meta-analysis. Neurocrit Care 10:253-256, 2009

7. Bruce J, Russell EM, Mollison J, Krukowski ZH: The measurement and monitoring of surgical adverse events. Health Technol Assess 5:1-194, 2001

8. Christian CK, Gustafson ML, Roth EM, Sheridan TB, Gandhi TK, Dwyer K, et al: A prospective study of patient safety in the operating room. Surgery 139:159-173, 2006

9. Deshpande JK: Cause and effect or conjecture? A call for consensus on defining "anesthesia-related mortality." Anesth Analg 112:1259-1261, 2011

10. Drake JM, Riva-Cambrin J, Jea A, Auguste K, Tamber M, Lamberti-Pasculli M: Prospective surveillance of complications in a pediatric neurosurgery unit. J Neurosurg Pediatr 5:544-548, 2010

11. Drake JM, Singhal A, Kulkarni AV, DeVeber G, Cochrane DD: Consensus definitions of complications for accurate recording and comparisons of surgical outcomes in pediatric neurosurgery. J Neurosurg Pediatr 10:89-95, 2012

12. Edomwonyi NP, Ekwere IT, Egbekun R, Eluwa B: Anesthesia-related complications in children. Middle East J Anaesthesiol 18:915-927, 2006

13. Fabri PJ, Zayas-Castro JL: Human error, not communica- 
tion and systems, underlies surgical complications. Surgery 144:557-565, 2008

14. Gonzalez LP, Pignaton W, Kusano PS, Módolo NS, Braz JR, Braz LG: Anesthesia-related mortality in pediatric patients: a systematic review. Clinics (Sao Paulo) 67:381-387, 2012

15. Huyette DR, Turnbow BJ, Kaufman C, Vaslow DF, Whiting BB, Oh MY: Accuracy of the freehand pass technique for ventriculostomy catheter placement: retrospective assessment using computed tomography scans. J Neurosurg 108:88-91, 2008

16. Jöhr M: [Risks in pediatric anesthesia. Causes and prevention of complications.] Anaesthesist 63:546-547, 2014 (Ger)

17. Keenan RL, Shapiro JH, Dawson K: Frequency of anesthetic cardiac arrests in infants: effect of pediatric anesthesiologists. J Clin Anesth 3:433-437, 1991

18. Lee M, Rezai AR, Chou J: Depressed skull fractures in children secondary to skull clamp fixation devices. Pediatr Neurosurg 21:174-178, 1994

19. Lenke LG, Newton PO, Sucato DJ, Shufflebarger HL, Emans JB, Sponseller PD, et al: Complications after 147 consecutive vertebral column resections for severe pediatric spinal deformity: a multicenter analysis. Spine (Phila Pa 1976) 38:119-132, 2013

20. Murat I, Constant I, Maud'huy H: Perioperative anaesthetic morbidity in children: a database of 24,165 anaesthetics over a 30-month period. Paediatr Anaesth 14:158-166, 2004

21. Saladino A, White JB, Wijdicks EF, Lanzino G: Malplacement of ventricular catheters by neurosurgeons: a single institution experience. Neurocrit Care 10:248-252, 2009

22. Singh GP, Prabhakar H, Bithal PK, Dash HH: A retrospective analysis of perioperative complications during intracranial neuroendoscopic procedures: our institutional experience. Neurol India 59:874-878, 2011

23. Tay CL, Tan GM, Ng SB: Critical incidents in paediatric anaesthesia: an audit of 10000 anaesthetics in Singapore. Paediatr Anaesth 11:711-718, 2001
24. Toma AK, Camp S, Watkins LD, Grieve J, Kitchen ND: External ventricular drain insertion accuracy: is there a need for change in practice? Neurosurgery 65:1197-1201, 2009

25. van Lindert EJ, Delye H, Leonardo J: Prospective review of a single center's general pediatric neurosurgical intraoperative and postoperative complication rates. J Neurosurg Pediatr 13:107-113, 2014

26. Vitali AM, Steinbok P: Depressed skull fracture and epidural hematoma from head fixation with pins for craniotomy in children. Childs Nerv Syst 24:917-925, 2008

\section{Disclosures}

The authors report no conflict of interest concerning the materials or methods used in this study or the findings specified in this paper.

\section{Author Contributions}

Conception and design: van Lindert. Acquisition of data: van Lindert, Arts, Blok. Analysis and interpretation of data: van Lindert. Drafting the article: van Lindert. Critically revising the article: all authors. Reviewed submitted version of manuscript: all authors. Approved the final version of the manuscript on behalf of all authors: van Lindert. Statistical analysis: van Lindert. Administrative/technical/material support: van Lindert, Arts. Study supervision: van Lindert.

\section{Correspondence}

Erik J. van Lindert, Department of Neurosurgery, Radboud University Medical Centre, Geert Groteplein-Zuid 10, PO Box 9101, Nijmegen 6500 HB, The Netherlands. email: erik. vanlindert@radboudumc.nl. 